\title{
Multi-Inputs and Multi-Outputs Mems Resonator for Complex Logic Operations
}

\author{
Sherif A. Tella and Mohammad I. Younis \\ Physical Science and Engineering Division \\ King Abdullah University of Science and Technology \\ Thuwal. Makkah Province, Saudi Arabia \\ sherif.tella@kaust.edu.sa, mohammad.younis@kaust.edu.sa
}

\begin{abstract}
Microelectromechanical systems MEMS resonatorbased computing devices have recently attracted significant attention due to their simplicity and potential toward energy efficient computing machines. Lately, there have been successful demonstrations of fundamental logic gates. However, the realization of complex multifunctional logic gates that require multi-input and multi-output lines have faced some obstacles, such as the interconnections between multiple resonators and the increase in device complexity due to the large required arrays of resonators. This paper demonstrates a 1:2 demux combinational logic gate from the first and the second vibrational modes of a single MEMS microstructure. The MEMS device consists of three connected inplane microbeams forming a $U$-shape. The microbeams can be electrostatically actuated individually or collectively.
\end{abstract}

Keywords - MEMS resonator, logic gates, vibrational modes, axial stress modulation, 1:2 demux

\section{INTRODUCTION}

There have been an increasing interest in the use of microelectromechanical systems (MEMS) as digital computing devices. Several fundamental digital logic gates, switches, and memory devices have been demonstrated based on the static and dynamic principles of MEMS microstructures[1]-[6]. An axially modulated clamped-guided arch resonator was used to experimentally demonstrate logic and memory devices [7]. Also logic gates (NOR, XOR and AND gates) were demonstrated using linear frequency modulation of the arch resonator. Despite successful demonstration of fundamental logic gates, the realization of complex combinational logic gates has posed a great challenge because most complex logic gates require multi-input and multi-output lines. In view of this challenges, Hafiz et al. [8] reported an alternative approach by cascading multiple electrothermally actuated arch resonators to demonstrate a 2:1 mux logic gates. Another approach was also reported in [9] based on a single parametrically actuated MEMS resonator to which mechanical oscillations at different frequencies were encoded into multiple channels of binary information to demonstrate universal logic gates with multibit logic functions. However, there are also obstacles to these alternative approaches such as the interconnections between the multiple resonators [10], the device complexity and the controllability over the frequency of operations. Toward these challenges, a novel approach was reported in [3] to demonstrate multi-input and multi-output complex combinational logic gates from a single MEMS microstructure. However, this approach is based on electrothermal actuation that makes the system not power efficient and also can lead to frequency drift due to changes in temperature.

In this paper, we demonstrate a 1:2 demux complex logic gate. The first and second modes of vibrations for the single MEMS microstructure are used as the operating frequency for the outputs. The proposed device can be actuated and switched electrostatically through the electrostatic force from the side beams. Thus, the proposed microstructure provides promising platform to demonstrate complex logic gates that require multiinput and multi-output lines.

\section{DESIGN PRINCIPLE}

The SEM image of the fabricated device are shown in Fig. 1. The device is fabricated by MEMSCAP [11] based on a twomask lithography process with a phosphorus dopant into the top surface of the silicon layer of the Silicon-On-Insulator (SOI) wafer. The design structure consists of three in-plane microbeams that form a single MEMS resonator. This structure has two side beams connected to an arch beam that its ends are guided by the tips of the side beams. The side beams are sandwiched between two electrodes that can be used to induce both tensile and compressive axial loads on the arch beam. This provides platforms to modulate the resonance frequency of the arch beam. These electrodes can also be used as sensing and actuating electrodes for the side beams as it will be demonstrated in the subsequent section. The dimensions of the three microbeams are listed in Table 1.

As experimentally demonstrated in [12], the stiffness and resonant frequencies of an arch beam can be tuned and controlled by applying either tensile or compressive axial loads and these can affect its static and dynamic behavior. The controllability and tunability of the frequency responses for the first and second modes of the single MEMS resonator are used to select the operating frequencies to implement the logic operation.

This publication is based upon work supported by the King Abdullah University of Science and Technology (KAUST) office of sponsored research OSR under Award No. OSR-2016-CRG5-3001 
TABLE 1: Dimensions of the microbeams

\begin{tabular}{|c|c|c|c|c|}
\hline Description & \multicolumn{2}{|c|}{$\begin{array}{l}\text { Side Straight Beams (A } \\
\text { and B) }\end{array}$} & \multicolumn{2}{|c|}{ Arch Beam } \\
\hline Length $(\mu \mathrm{m})$ & $L_{1}$ & 100 & $L_{2}$ & 300 \\
\hline Width $(\mu \mathrm{m})$ & $h_{1}$ & 2 & $h_{2}$ & 2 \\
\hline Depth $(\mu \mathrm{m})$ & $b_{1}$ & 25 & $b_{2}$ & 25 \\
\hline $\begin{array}{c}\text { Initial Curvature } \\
(\mu \mathrm{m})\end{array}$ & & 2 & $a$ & 2 \\
\hline $\begin{array}{c}\text { Gap between the } \\
\text { beams and } \\
\text { electrodes }(\mu \mathrm{m})\end{array}$ & $d$ & $g$ & 3 \\
\hline
\end{tabular}

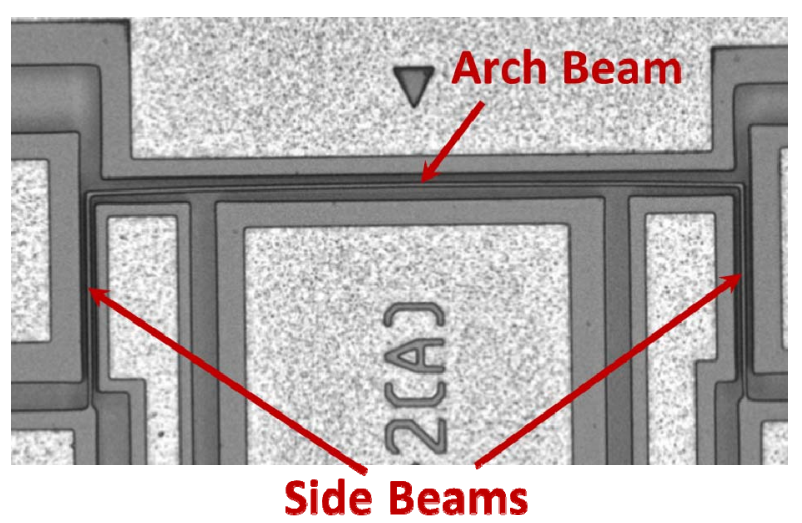

Fig. 1: A scanning electron microscope (SEM) image of the fabricated device.

\section{EXPERIMENTAL SETUP}

For the experimental characterization of the device, Network Analyzer (E5071C) is used as shown in Fig. 2. In demonstrating the complex logic gate, two outputs are required and the sensing electrodes of one of the side beam and the arch beam are used as the outputs while the corresponding driving electrodes as shown in Fig. 2 are used to excite the structure into the required modes of vibrations. The tensile and the compressive electrodes of the other side beam are used as input and selector, respectively. The electrostatic actuation for the driving electrodes consists of an in-built AC signal from the Network Analyzer superimposed with a DC voltage from DC supply. The generated vibrations due to the electrostatic actuation induces motional currents in the sense electrodes and these form the output currents that is amplified by the low noise amplifier (LNA). The outputs from LNA are connected to the input ports of the network analyzer for $S_{21}$ transmission measurements. For the demonstrated logic operation, the arch beam and the side beam are biased with $15 \mathrm{~V}$ DC voltage and AC signal of $-20 \mathrm{dBm}(22.361 \mathrm{mVrms})$ at the pressure of 500 $\mathrm{mTorr}$ and room temperature.

\section{RESULTS AND DISCUSSION}

The first step toward performing the logics is to study the tunability of the first two modes of the compound structure, which will be the basis of operations for the subsequent operations. Fig. 3 (a) shows the modes of vibrations for the two operating modes as obtained from a finite element analysis using
COMSOL. Fig. 3(b) and (c) show the tunability of both modes as the input and selector electrodes are varied with DC voltages. The tunability of the modes are only carried out for the input and the selector electrodes that are required to perform the logic operation as shown in the truth table of Table 2. The resonant frequencies of both modes are obtained to be $95.49 \mathrm{kHz}$ and $105.6 \mathrm{kHz}$ respectively. As shown in Fig. 3(b), the resonant frequency of the first mode decreases steadily as the voltage increases in both cases when the selector only is in ON state and when both input and selector are in ON states. The resonant frequency of the second mode also shows similar result when only the input is in ON state (see Fig. 3(c)) however, the resonant frequency shows a different qualitative result when only the selector is in the ON state. The frequency decreases until it gets to the critical point after which it increases as the voltage increases. As reported in [13], this observed phenomenon is due to the competing effect between the initial curvature of the arch beam and the applied tensile axial load from the selector.

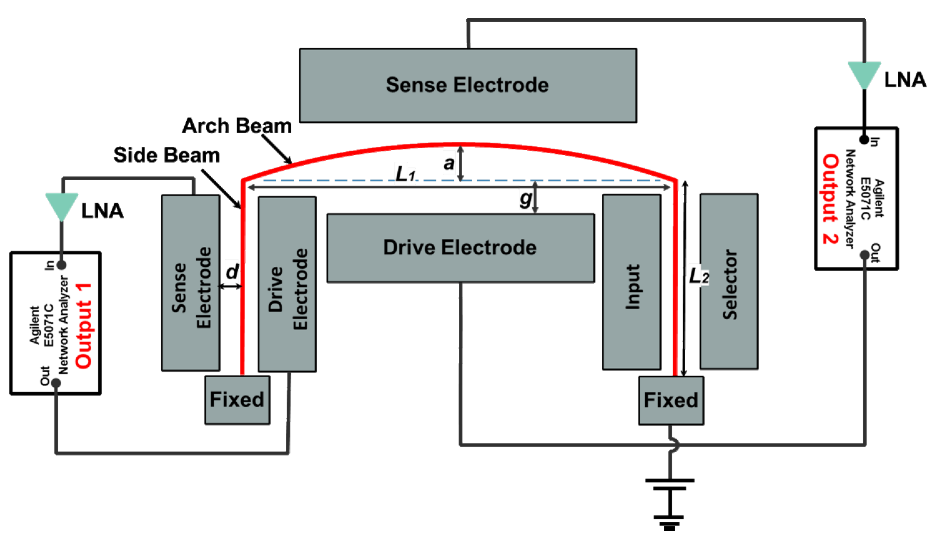

Fig. 2: Schematic of the experimental setup.
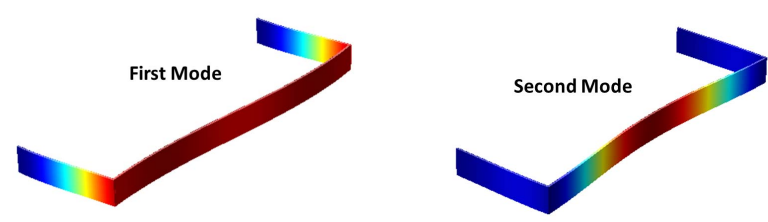

(a) 


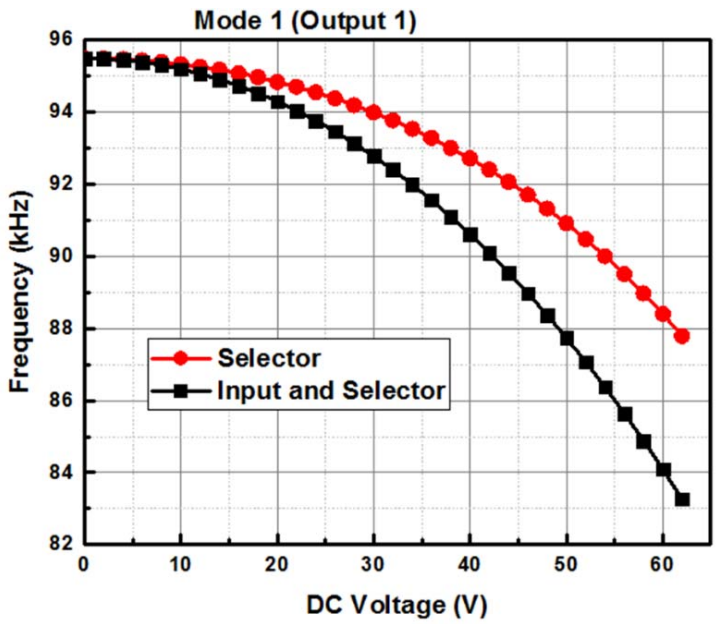

(b)

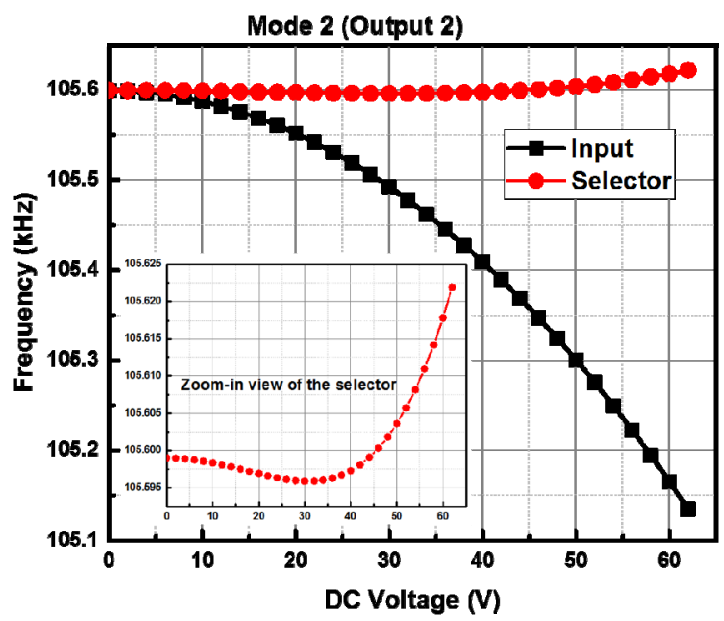

(c)

Fig. 3: (a) First and second mode of vibrations. Measurement of resonant frequency variation for (b) Mode 1 (Output 1) when only the selector is in ON state and when both input and selector are in ON state. (c) Mode 2 (Output two) when only input and only output are in ON state.

\section{A. 1:2 DEMUX LOGIC OPERATION}

A demux is an electronic device that takes a single input line, which is routed, to one of several digital output lines. A demux of $2^{\mathrm{n}}$ output lines has $\mathrm{n}$ selectors. The 1:2 demux demonstrated in this work takes a single input from the input electrode and routes it to one of the two output lines (sense electrodes) with the help of the selector. The truth table is shown in Table 2. As indicated in the truth table, when the selector is in the OFF state and the input line is in the ON state (i.e. $\mathrm{S}=0$ and $\mathrm{IN}=1$ ), the input line is routed to output 2, however, when the selector is in the ON state and the input line is also in the $\mathrm{ON}$ state (i.e. $\mathrm{S}=1$ and $\mathrm{IN}=1$ ), the input line is routed to output 1 . The corresponding voltages for the OFF and the ON states for both the selector $0(1)$ and the input $0(1)$ are $0 \mathrm{~V}$ and $30 \mathrm{~V}$, respectively. The $\mathrm{S}_{21}$ transmission measurement for both output lines are shown in Fig. 4(a) and (b). Using the measured values obtained from the tunability of both modes, the operating frequencies for output 1 and output 2 are selected as $92.8 \mathrm{kHz}$ and $105.48 \mathrm{kHz}$, respectively. Activating a $30 \mathrm{~V}$ on the input and $0 \mathrm{~V}$ on the selector, the resonance frequency of mode 2 is tuned to $105.48 \mathrm{kHz}$, which is the frequency of operation for output 2 . Likewise when applying $30 \mathrm{~V}$ on input and selector, the resonance frequency of mode 1 is tuned to $92.80 \mathrm{kHz}$ as shown in Fig. 4(a).

Table 2: Truth table of 1:2 Demux logic gate.

\begin{tabular}{|c|c|c|c|}
\hline Selector & Input & \multicolumn{2}{|c|}{ Outputs } \\
\hline S & IN & Out 1 & Out 2 \\
\hline 0 & 0 & 0 & 0 \\
\hline 0 & 1 & 0 & 1 \\
\hline 1 & 0 & 0 & 0 \\
\hline 1 & 1 & 1 & 0 \\
\hline
\end{tabular}

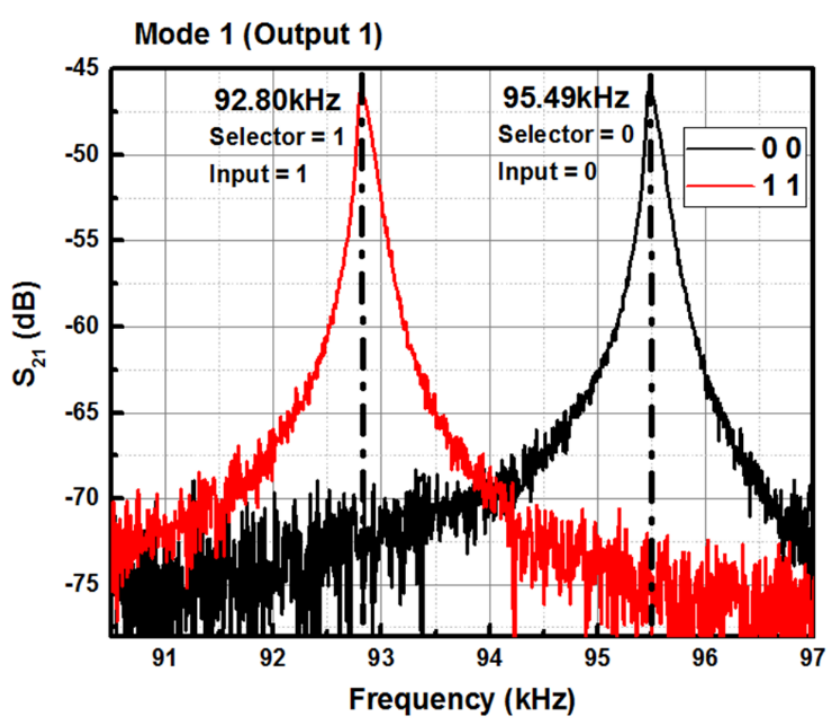

(a)

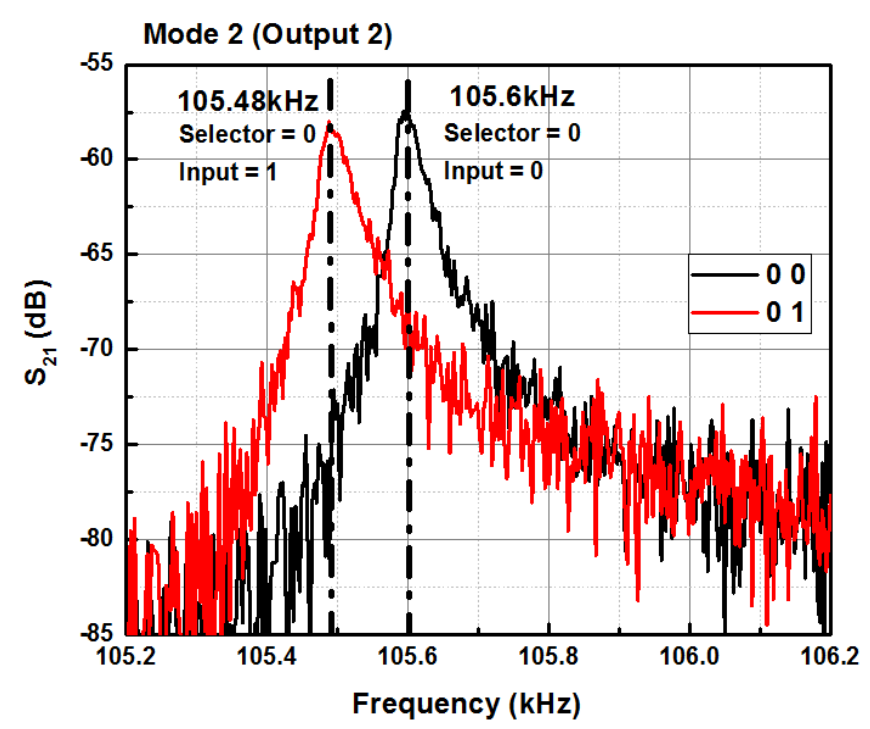

(b) 
Fig. 4: (a) Frequency response of mode 1 for Output 1. (b) Frequency response of mode 2 for Output 2.

\section{CONCLUSION}

This paper has demonstrated the design and operation of a multi-inputs and multi-outputs MEMS resonator for complex logic gate. The first and second modes of vibrations for the single MEMS microstructure are used as the operating frequency for the outputs. The principle of operation is based on modulating the resonance frequencies of the vibrational modes through axial electrostatic loads from the side beams. Thus, the proposed microstructure provides promising platform to demonstrate complex multifunctional logic gates that require multi-input and multi-output lines with improved energy efficiency.

\section{REFERENCES}

[1] W. J. Venstra, H. J. R. Westra, and H. S. J. Van Der Zant, "Mechanical stiffening, bistability, and bit operations in a microcantilever," Applied Physics Letters, vol. 97, no. 193107, pp. 1-4, 2010.

[2] J. Wenzler, T. Dunn, T. Toffoli, and P. Mohanty, "A Nanomechanical Fredkin Gate," Nano Letters, vol. 14, pp. 89-93, 2013.

[3] S. A. Tella, N. Alcheikh, and M. I. Younis, "A single MEMS resonator for reconfigurable multifunctional logic gates," Journal of Micromechanics and Microengineering, vol. 28, no. 095002, pp. 1-9, 2018.

[4] A. Yao and T. Hikihara, "Counter operation in nonlinear micro-electro-mechanical resonators," Physics Letters A, vol. 377, no. 38, pp. 2551-2555, 2013.

[5] A. Yao and T. Hikihara, "Logic-memory device of a mechanical resonator," Applied Physics Letters, vol.
105, no. 123104, pp. 1-5, 2014.

[6] C. Chu, W. Shih, S. Chung, H.-C. Tsai, T.-K. Shing, and P.-Z. Chang, "A low actuation voltage electrostatic actuator for RF MEMS switch applications," Journal of Micromechanics and Microengineering, vol. 17, pp. 1649-1656, 2007.

[7] A. Al Hafiz, S. Tella, N. Alcheikh, H. Fariborzi, and M. I. Younis, "Axially Modulated Clamped-Guided Arch Resonator for Memory and Logic Applications," in Proceedings of the ASME Design Engineering Technical Conference, 2017, pp. 1-6.

[8] M. A. A. Hafiz, L. Kosuru, M. I. Younis, and H. Fariborzi, "A 2: 1 MUX based on multiple MEMS resonators," in Procedia Engineering, 2016, vol. 168, pp. 1642-1645.

[9] I. Mahboob, M. Mounaix, K. Nishiguchi, A. Fujiwara, and H. Yamaguchi, "A multimode electromechanical parametric resonator array," Scientific Reports, vol. 4, no. 4448, pp. 1-8, 2014.

[10] I. Mahboob, E. Flurin, K. Nishiguchi, A. Fujiwara, and H. Yamaguchi, "Interconnect-free parallel logic circuits in a single mechanical resonator," Nature Communications, vol. 2, pp. 197-198, 2011.

[11] MEMSCAP, "SOIMUMPs." [Online]. Available: http://www.memscap.com/products/mumps/soimumps. [Accessed: 20-Feb-2019].

[12] S. Tella, N. Alcheikh, and M. I. Younis, "Electrothermally Actuated Microbeams with Varying Stiffness," in Proceedings of the ASME Design Engineering Technical Conference, 2017, pp. 1-6.

[13] S. A. Tella, A. Z. Hajjaj, and M. I. Younis, "The effects of initial rise and axial loads on MEMS arches," Journal of Vibration and Acoustics, Transactions of the ASME, vol. 139, no. 040905, pp. 1-8, 2017. 